\title{
Whole Genome Resequencing of Jordanian Awassi Rams (Ovis aries) Using Hiseq Sequencing Technology: The First Step Towards Sheep Genomic Selection
}

\author{
N. Haddad ${ }^{1}$, H. Migdadi, ${ }^{1,2}$, R. Al-Atiyat ${ }^{3}$, K. Jawasreh ${ }^{4}$, S. Awabdeh ${ }^{1}$, \\ W. Obeidat ${ }^{1}$, R. AlOmari ${ }^{1}$, M. Aldamra ${ }^{1}$, H. Ababneh ${ }^{1}$, M.J. Tabbaa ${ }^{5}$, \\ M. Brake ${ }^{6}$ and M. Farooq ${ }^{7,8,9}$ \\ ${ }^{1}$ National Agricultural Research Center, Baqa, Jordan \\ ${ }^{2}$ Plant Production Department, College of Food and Agriculture Sciences, \\ King Saud University, Riyadh, Saudi Arabia \\ ${ }^{3}$ Department of Animal Science, Agriculture Faculty, Mutah University, \\ Karak, Jordan \\ ${ }^{4}$ Department of Animal Production, Jordan University of Science and \\ Technology, Irbid, Jordan \\ ${ }^{5}$ Department of Animal Production, School of Agriculture, University of \\ Jordan, Amman, Jordan \\ ${ }^{6}$ Department of Science, Jerash University, Jordan \\ ${ }^{7}$ Legume Research Group, Plant Production Department, College of Food \\ and Agricultural Sciences, King Saud University, Riyadh, Saudi Arabia \\ ${ }^{8}$ Department of Crop Sciences, College of Agricultural and Marine Sciences, \\ Sultan Qaboos University, Al-Khoud, Oman \\ ${ }^{9}$ Department of Agronomy, University of Agriculture, Faisalabad, Pakistan
}

Corresponding author: H. Migdadi

E-mail: h.migdadi@gmail.com

Genet. Mol. Res. 19 (2): gmr 18577

Received February 10, 2019

Accepted April 30, 2020

Published June 30, 2020

DOI http://dx.doi.org/10.4238/gmr18577

ABSTRACT. We report the whole ram genome of Jordanian Awassi (Ovis aries), screened using the power of Illumina HiSeq sequencing technology, providing insights into the ram's genomic structure. Generated data will help in the assessment of naturally occurring genetic variation and population structure of this sheep breed using different structure variation markers. Also, it will help in the established markertrait associations that can be used in marker-assisted breeding for qualitative and quantitative productive traits. A total of 23,812,247 single 
nucleotide polymorphism (SNPs) were identified, of which 177,117 $(0.74 \%)$ were in the coding regions, as well as 3.77 million insertions/deletions and 3357 frame-shifting mutations in the coding region. The re-sequencing revealed 38,900 structure variation types distributed along genome, including 45 insertions and 16,643 deletions types. Also, there were 13,689 copy number variations, of which 3743 were up-regulated, and 9946 were down-regulated. These results will help in describing SNPs and the distribution of structural variations types used in genetic mapping and breeding programs of sheep breeds.

Key words: Ovis aries; Sheep; SNPs; Insertions / deletions; Copy number of variations

\section{INTRODUCTION}

Awassi sheep (Ovis aries) is the dominant fat tail sheep breed in the Mediterranean countries, including Jordan. It has many unique characteristics, including acceptable performance under harsh conditions and its preferred meat quality by Mediterranean consumers (Tabbaa et al., 2001). Also, it has various contributions to the vast majority of the livelihood of the Bedouins, making it a superior sheep breed compared to imported sheep. Awassi sheep in Jordan are well characterized for their carpet wool, body weight, and morphological characteristics at different ages (Tabbaa, 1998 and 2003). Semen of Awassi rams and reproductive characteristics of Awassi ewes have been characterized (Tabbaa et al., 2006 and 2008).

Over the last three decades, reports on releasing and developing sheep genome have appeared. Crawford et al. (1995) showed a detailed genetic linkage map of sheep covering 2070 cM of the sheep genome. Two years later, Barendse et al. (1997) reported a new genetic linkage sheep map with 446 highly informative markers. In 2001, a sheep genic linkage map was generated with more than 1000 loci (Maddox et al., 2001). In the year 2009, the sheep genome announced and identified expressed genes associated with the biology of the rumen and lipid metabolism (Jiang et al., 2014). In the same year, a linkage map for males from a single-family of Awassi-Merino backcross progeny reported (Raadsma et al., 2009). Studying the genome of the male sheep (Ram) is a characteristic of interest to sheep breeders and geneticists.

Evidence from Mediterranean archaeozoological sites suggested that sheep were first domesticated in the nearby Arabian Peninsula in the Fertile Crescent region in Southwest Asia 11,000 years ago (Zeder, 2008) and spread out from these domestication centers into the world during the subsequent few thousand years (Chessa et al., 2009). Humans reared sheep for s meat before wool and milk were used (Chessa et al., 2009). Following their domestication and selection, they have been adapted to a diverse range of environments. The implementing of quantitative genetics and the use of artificial insemination to prioritize superior rams has resulted in a vast increase of genetic gain in sheep breeding (Kijas et al., 2012)

Various types of molecular genetic evidence show that Jordan Awassi has common haplotype groups (B and A) and group C with Saudi, Kuwaiti, Egypt, and Yemen sheep considering mitochondrial DNA (mtDNA) and microsatellite-based evidence (Al-Atiyat et al., 2018; Jawasreh et al., 2018). The National Agricultural Research Center in Jordan set up to do whole genome sequencing for 100 Jordanian native species, including plants, animals, and insects. The main aims were to facilitate access to and use data incorporated in the international domain (NCBI) and to find genetic variants in the populations studied. The project started sequencing the native honeybee; Apis mellifera syriaca (Wallberg et al., 2014; Haddad et al., 
2016), the Oriental Hornet, Vespa orientalis (Haddad et al., 2017), and in 2019 three genomes were completed, which were the Awassi ram, Awassi ewe, and ancient Jordanian olive.

Jordan Awassi revealed a high level of genetic variability based on molecular markers (Al-Atiyat et al., 2012). Jawasreh et al. (2011) reported for the first time, the new Sagri separate Awassi strain reported to be of distinct characteristics found in Jordan Valley and started to distribute in Jordanian governorates. In Jordan, studies on Awassi sheep involved genetic diversity and genetic structure and its relatedness with other sheep breeds (Al-Atiyat et al., 2014; Jawasreh et al., 2018), genetic parameters of some essential genes and their association with some economic traits (Jawasreh et al., 2007, 2010, 2012, 2017, 2018 and 2019).

National sheep breeding strategy in Jordan is based on ram selection, including an advanced genotyping test for economic traits and identified day blindness mutation found in imported improved Awassi (Jawasreh et al., 2007, 2010, 2012 and 2017). Genetic mutation of reported Microtia (earless) controlled by GATA-6, was also investigated using genome-wide association (GWAS) (Jawasreh et al., 2016). Advanced genome selection techniques will be required to speed-up Awassi sheep improvement, putative selection, understanding disease inheritance, and get insights into the genetic control of milk, meat, and wool production. Overall, increasing our knowledge of Awassi sheep evolution and breeding was the principal motivation for the current resequencing of Jordanian Awassi (Ovis aries) Ram whole genome.

\section{MATERIAL AND METHODS}

\section{Animal Samples}

Animal care and use approved all experimental protocols involving animals with the ethical approval of relevant national legislation (Approval Code No. (G/6) 2006): Instructions and conditions for the acquisition of test animals and testing them).

Whole-blood samples $(10 \mathrm{~mL})$ collected from 13 rams from three main Awassi sheep producing areas in Jordan kept in the freezer till DNA extraction. Genomic DNA extracted using the DNA purification kit (Promega, Wisconsin, USA) following instructions of the manufacturer. Genomic DNA was fragmented, and after electrophoresis, DNA fragments of desired length was gel purified. Adapter ligation and DNA cluster preparation were performed and subjected to the Ilumina Hiseq 2500 sequencing system (Figure 1).

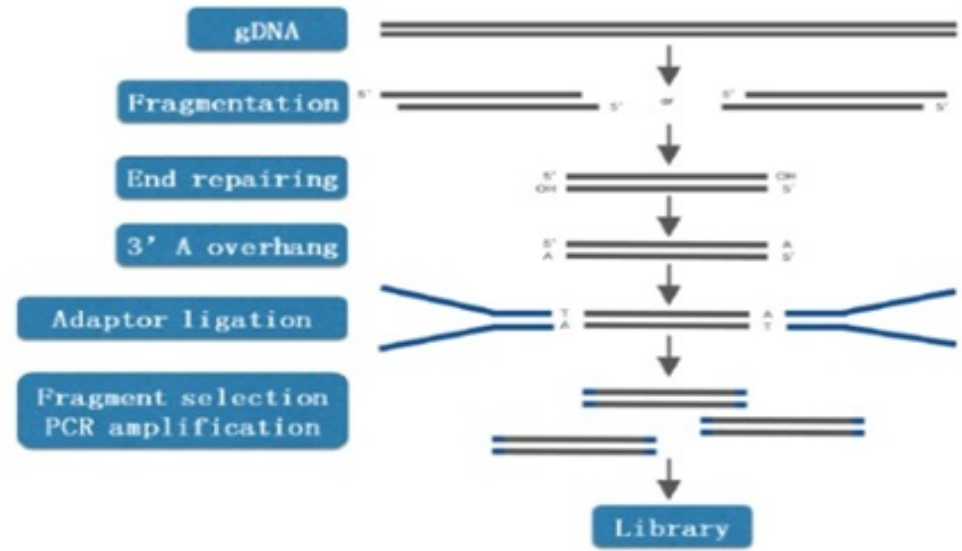

Figure 1. The detailed workflow of the "Quantification Pipelines" module in Panel used in the whole genome resequencing of Jordanian Awassi sheep. 


\section{The pipeline of Bioinformatics Analysis}

The sequencing data generated from the Illumina pipeline started with the removing of the adapter sequence, and the low-quality reads discarded to produce clean data. The next step was to use Burrows-Wheeler Aligner (http://bio-bwa.sourceforge.net/) (Li and Durbin, 2009) for aligning reads to the reference sequence. BAM format files used to store the alignment information. After fixing mate-pair information, adding read group information, and marking duplicate reads caused by polymerase chain reaction, variant calling, and detecting of Single Nucleotide Polymorphisms (SNPs) and small Insertion/Deletions (InDels) by GATK software (https://www.broadinstitute.org/gatk/) (McKenna et al., 2010). BreakDancer software (http://breakdancer.sourceforge.net/) (Chen et al., 2009) used for identification of Structure Variants (SVs) and Copy Number Variants (CNVs). At each stage of the analysis, quality control applied for the alignment and the called variant (Figure 2).

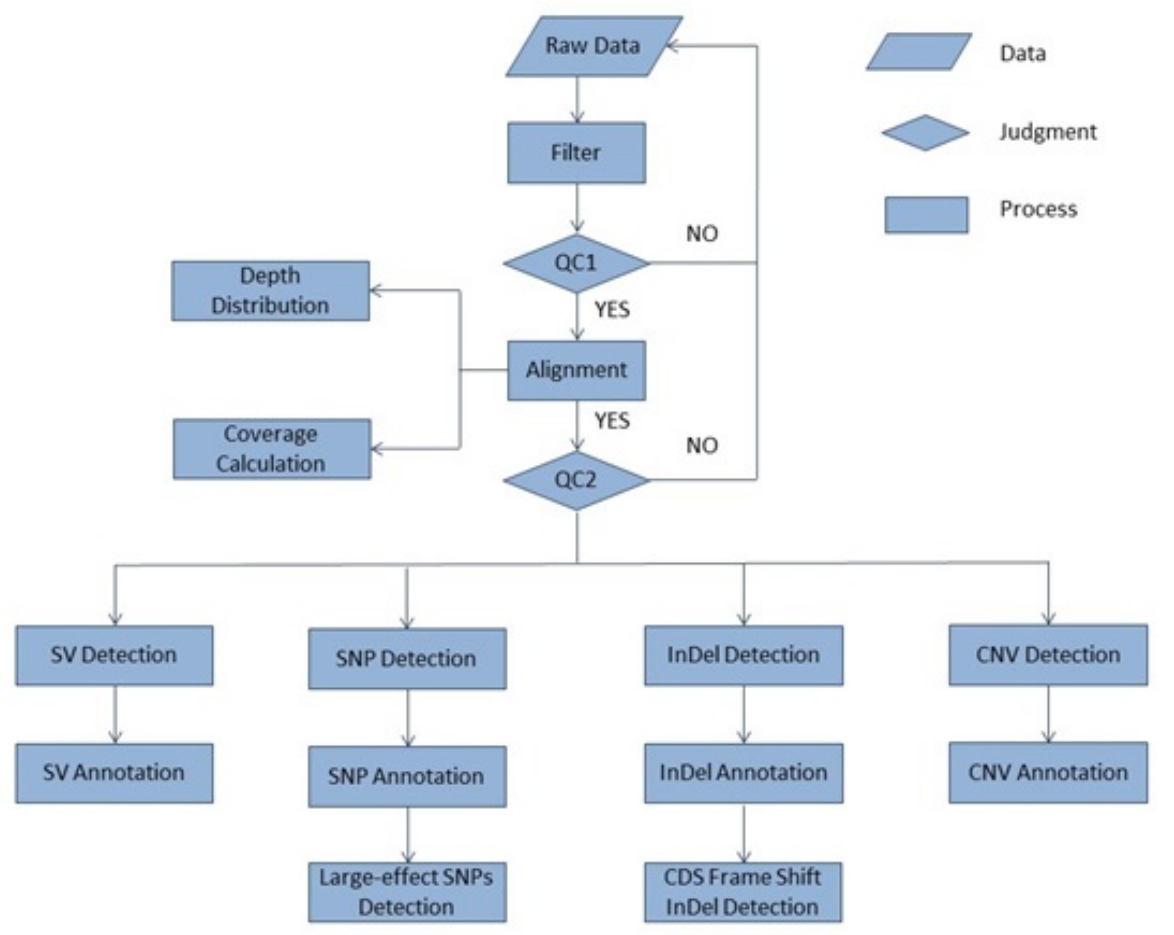

Figure 2. The pipeline of Standard Bioinformatics Analysis. The pipeline based on the family of SOAP software.

\section{Filter Reads}

Raw reads contain adapters, unknown or low-quality bases discarded. The parameters of SOAP nuke (unreleased) software for this project is: "-n $0.05-120-\mathrm{q} 0.2-\mathrm{G}$ Q 2". The filter steps included removing reads more than half of the bases' qualities are less than five and get clean reads. 
Alignments

Sequencing reads aligned using BWA software (http://biobwa.sourceforge.net/bwa.shtml) (Li and Durbin, 2009) to the reference genome. The short sequence aligns against long the reference providing the output of the sequence in SAM (Sequence Alignment/Map) format. Picard-tools (v1.118) were used to sort the SAM files by coordinate and converted them to BAM files. Genome Analysis Toolkit (GATK) ignored duplicate reads in later analyses. Picard tools software (v1.118) was also used to mark these duplicates.

\section{SNP and Short InDel Detection}

Single Nucleotide Polymorphisms (SNPs) and short InDels in our pipeline detect using GATK. After the identifying of SNPs or/and InDels, we use the programs developed by BGI to do annotation and classification of SNPs in gene regions. It provides statistics to present the distributing

\section{Structure variation (SV) Detection}

The read-pair method used to detect the structural variation. We use BreakDancer/CREST to detect SVs. A detailed description of the method used by BreakDancer found at http://breakdancer.sourceforge.net (Chen et al., 2009). Using reference genome our reads disturbed, which could be because of existing of deletions or insertions between the two mapped reads. The result is a list of structure variations detected at the whole genome level.

\section{Copy Number Variation Detection}

According to the method of CNVs detection (Zheng et al., 2011), based on the result of SOAP alignment, the depth of each base calculated and standardized by the mean depth of its chromosome to calculate the Copy Number Variation. Based on this theory, CNVs detected according to standard criteria, where, based on the base depth of each fragment, $\mathrm{P}$-value $\leq 0.35$ and mean depth $<0.5$ or $>2.0$ for probability to be CNV.

The genome raw data sequences of Jordanian Awassi sheep have been deposited in the NCBI under SRA (sequence reads archives) under the following accession numbers: PRJNA574879. SRA records will be accessible with the following link after the release date: https://www.ncbi.nlm.nih.gov/sra/PRJNA574879.

\section{RESULTS}

\section{Sequencing Assessment}

For ram samples, libraries with 64 paired-end sequencings with an insert size around $300 \mathrm{bp}$ constructed. The resequencing yielded $100.09 \mathrm{G}$ as clean databases in total. To ensure quality, the raw data changed by deleting the adapter pollution in reads and then the reads, which 1,929,009 contain over 50\% low-quality bases (quality value $\leq 12$ ) 
removed. The summaries of data and quality control shown in Table (1) and Figures 3 and 4. The Q20 base rate of each lane is above $98.73 \%$, so the data quality is very high.

Table 1. Summary of the sequencing results data clean reads in the Jordanian Awassi ram
Sheep.
\begin{tabular}{lc} 
\\
Sequence & Value \\
\hline GC rate & $43.35 \%$ \\
Q20 rate & $98.73 \%$ \\
Q30 rate & $93.95 \%$ \\
Reads & $1000.95 \mathrm{Mb}$ \\
Bases & $100.09 \mathrm{~Gb}$ \\
Clean Data/Raw & $94.63 \%$ \\
\hline
\end{tabular}

$\mathrm{Mb}$ and $\mathrm{Gb}$; mega and giga base pairs.

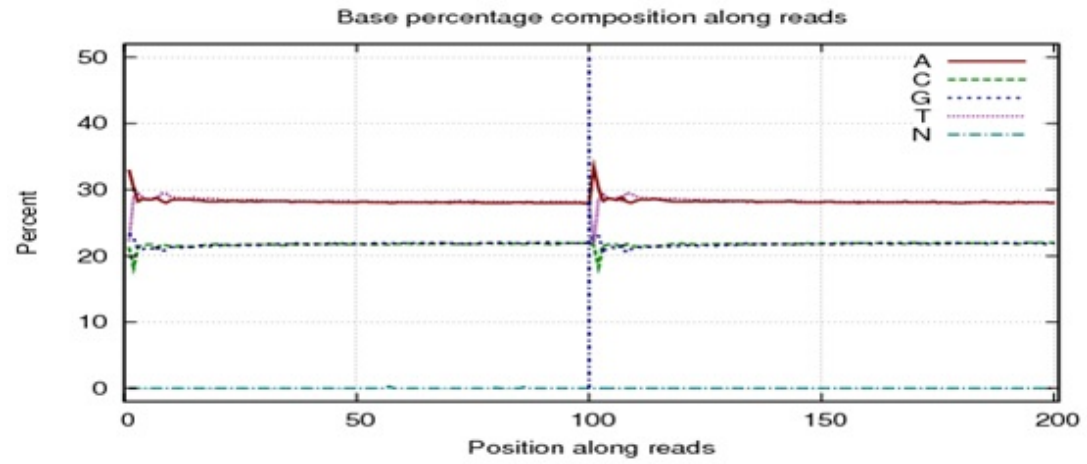

Figure 3. Distribution of base composition on clean reads, X-Axis positions, along with reads. Y-Axis means the base content rate. A curve overlapped with the $\mathrm{T}$ curve while the $\mathrm{G}$ curve overlapped with the $\mathrm{C}$ curve except for the first several bp positions (For Illumina sequencing platform, the random hexamer-primer used to synthesize the cDNA could cause PCR bias. So there are significant fluctuations in the first several bp positions along with reads, which is a typical situation). If an abnormal condition happens during sequencing, it may show an unbalanced composition

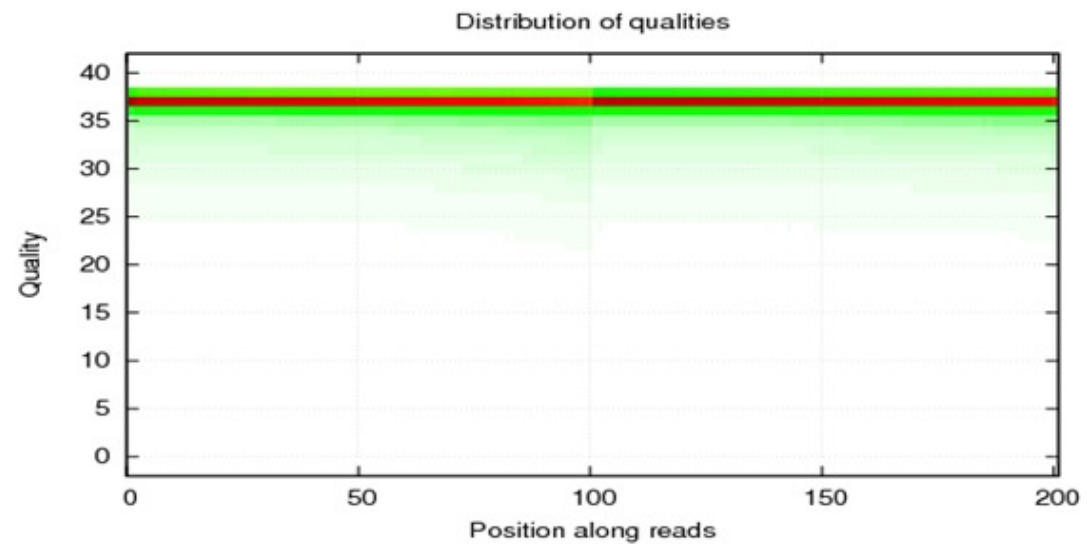

Figure 4. Distribution of base quality on clean reads, $\mathrm{X}$-axis positions along with reads. Y-axis is quality value. E8, 181,200ach dot in the image represents the quality value of the corresponding position along with reads. If the percentage of the bases with low quality $(<20)$ is very high, then the sequencing quality of this lane is inadequate. 
The reference genome, Sheep. Fa (Jiang et al., 2014) has taken for this project. The genome size is 2,584,832,510 bp and the effective size is 2,560,768,911 bp. BurrowsWheeler Aligner (BWA) software used for sequence reads alignment to the reference genome. The mapping rate and the final effective mapping depth in different accessions was 99.28\%, and 36.32 (Figure 5). Figures 6 and 7 show the distribution of sequencing depth and its cumulative depth besides the insert size distribution based on the result of the alignment.

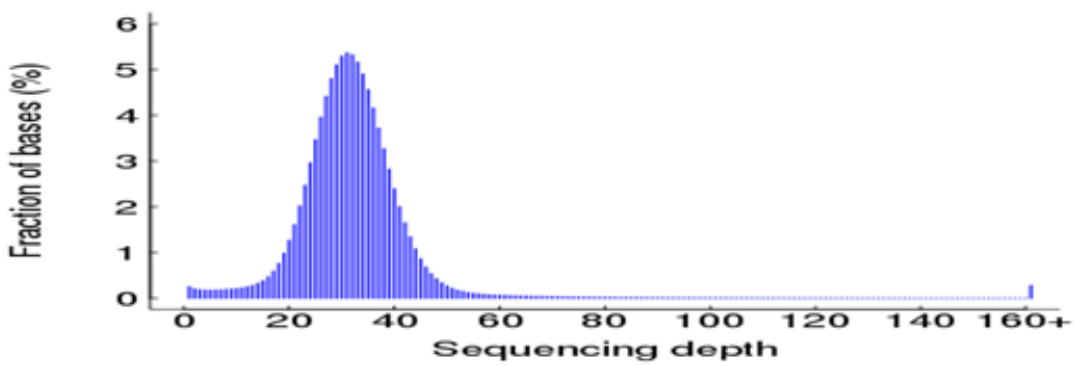

Figure 5. Sequencing depth histogram. The X-Axis is the sequencing depth, and Y-axis means the rate of the sequencing depth.

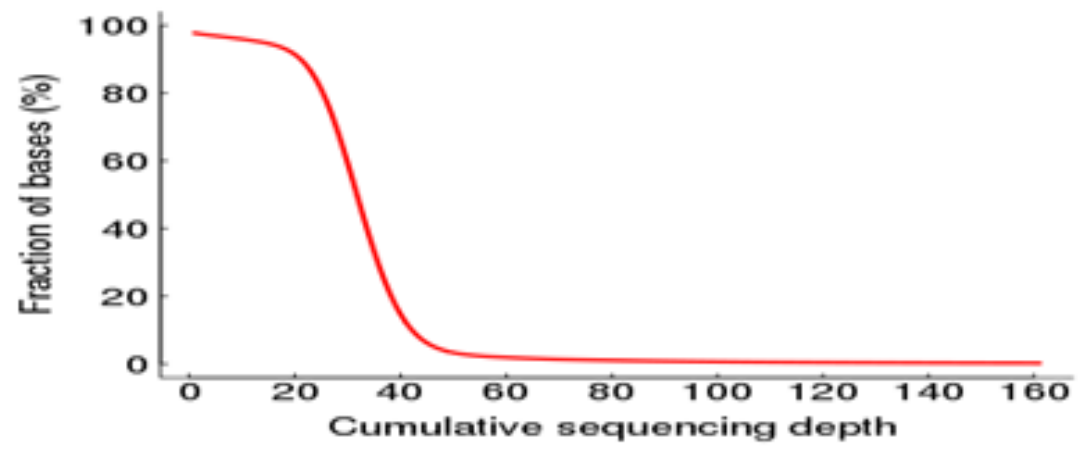

Figure 6. Distribution of cumulative sequencing depth. The X-Axis is the cumulative sequencing depth, and $\mathrm{Y}$ axis means the rate of the cumulative sequencing depth.

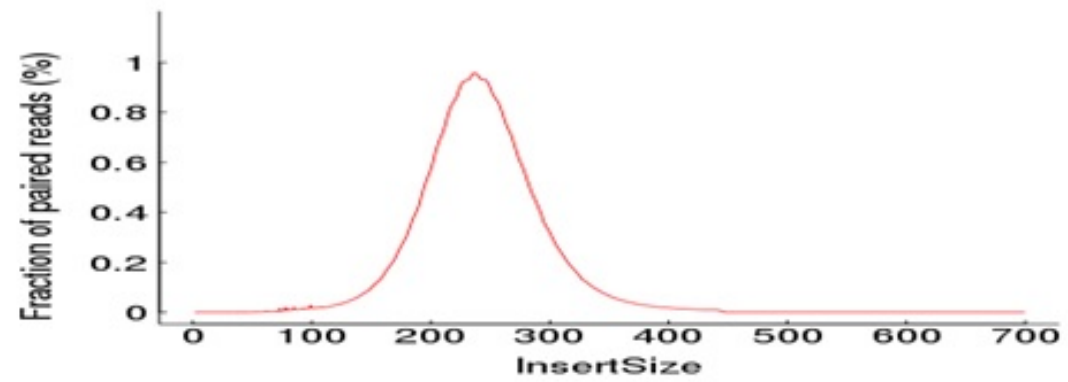

Figure 7. Insert size Distribution of Paired Reads. The X-axis represents the insert size of paired reads, and Yaxis shows the fraction of paired reads with an insert size. 


\section{SNP detection and annotation}

SNPs are small differences but with great impact on the variation of genomes and the biological traits. Based on the consensus sequence (all SNP we got), the polymorphic loci between the identified genotype and the reference can filter, and a high fidelity SNP data set generated (Table 2). 23,812,247 SNPs among this sheep genome, of which 177,117 $(0.74 \%)$ SNPs in the coding regions identified. The SNP results listed as CDS, exon, and genes. The distribution of SNP in the reference genome presented in Figure 8.

Table 2. Summary of genome-wide SNPs and the annotation distribution in
the Jordanian Awassi ram Sheep.
\begin{tabular}{lc}
\hline SNP distribution & Value \\
\hline SNP & $20,039,103$ \\
Homozygous & $2,403,946$ \\
Heterozygous & $17,635,157$ \\
Synonymous CDS & 161,420 \\
Nonsynonymous CDS & 107,116 \\
Exons & 842,118 \\
genes & $8,181,200$ \\
mRNA & $23,235,273$ \\
\hline
\end{tabular}

CDS: Coding sequence.

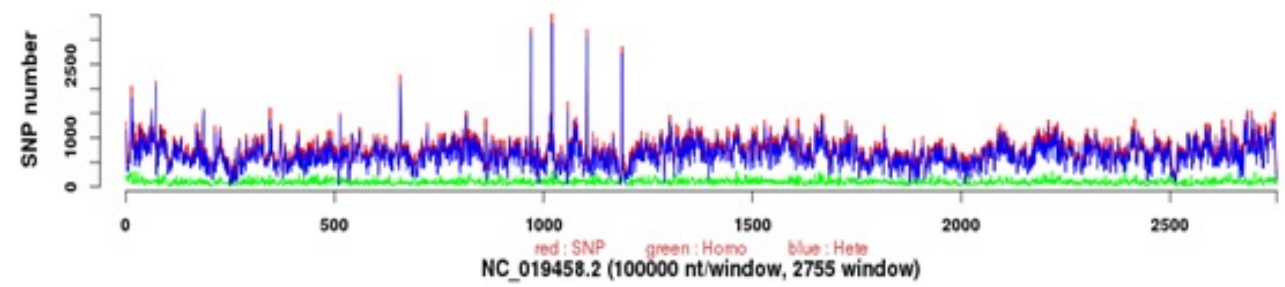

Figure 8. The distribution of SNPs in genome NC_019458.2. The X-axis represents the window numbers, and SNP numbers of the Y-axis mean the number of SNPs in each window.

In this project, 89,956 synonymous and 76,120 non-synonymous SNPs were annotated in this sheep genome. We went further to analyze the distribution of so-called large-effect SNPs, which are predicted to have a potentially disabling effect on gene function. The analysis showed that 1,035 SNPs were expected to induce premature stop codons, a 1,213 to disrupt splicing donor or acceptor sites, a 2,448 to alter initiation methionine residues, and 366 SNPs to remove the annotated stop codons, resulting in longer open reading frames.

\section{Short InDel detection and annotation}

The Genome Analysis Toolkit (GATK) was used to detect InDels, and the InDels result outputted in 'vcf' format. 3,769,565 InDels generated and distributed as 1,840,556 insertions, and 1,929,009 were deletions (Table 3 and Figure 9). After InDels identified, we annotated the result of InDel (Table 4). InDel annotation can reveal more details about InDels in some specific DNA regions, in the coding region. The effects of frame-shifting (e.g., 1-, 2-, 4-, 5-, 7-, 8-, 10-bp.) short InDels more significantly explain the different trait than 
those of non-frame-shift (e.g., 3-, 6-, 9-bp.) ones, so we further calculated the frame-shift InDel based on the InDel annotation result (Table 3).

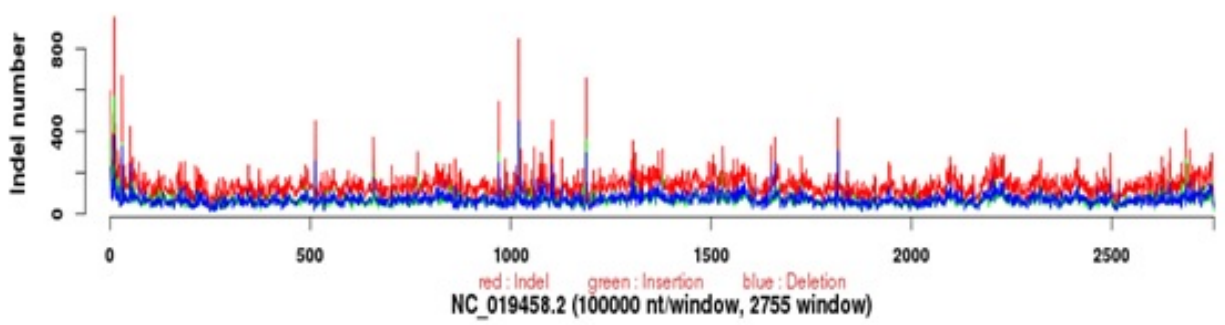

Figure 9. The distribution of InDel in genome NC_019458.2. 'InDel number' means the number of InDels in each window.

Table 3. Insertion and Deletion, annotation, distribution and frame shift mutation in CDS in the genome of Jordanian Awassi ram Sheep.

\begin{tabular}{lc}
\hline Insertion and Deletion & Value \\
\hline InDel & $3,769,565$ \\
Insertion & $1,840,556$ \\
Deletion & $1,929,009$ \\
CDS & 4,087 \\
C- gene segment & 3,182 \\
V- gene segment & 17,961 \\
cDNA match & 187,524 \\
Exon & 523,983 \\
Gene & 11,654 \\
mRNA & 483,879 \\
Frame-shift Mutation CDS & 3,357 \\
3X-shift Mutation CDS & 705 \\
3X-shift Mutation CDS Phase 0 & 234 \\
3X-shift Mutation CDS PhaseNo0 & 471 \\
\hline *CDS: Coding Sequence &
\end{tabular}

\section{Structure copy number variation detection and annotation}

Structure variation is an important difference among individuals of the same species. The exist of structural variation between the individual sequences and the reference; the alignment might not match. Such unusual paired-end alignment can thus use for detecting structure variation. The structural variation detected includes deletion, insertion, duplication, inversion, and transposition. $42.8 \%$ were deletion type of SV. The SV results presented in Table 4 and Figure 10.

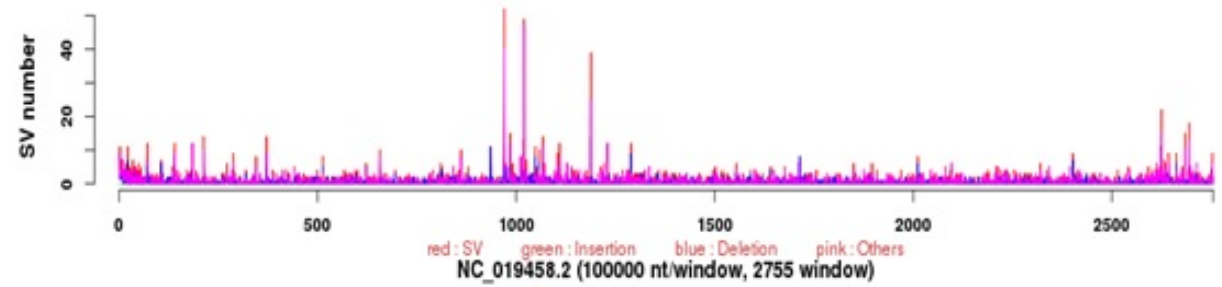

Figure 10. The distribution of structure variants (SV) in genome NC_019458.2. X-axis Y-axis: 'SV number' means the number of SVs in each window. 
Table 4. Structure variation annotation, distribution in the genome of Jordanian Awassi Sheep rams.

\begin{tabular}{ll}
\hline Structure variation & Value \\
\hline SV & 38,900 \\
Insertion & 45 \\
Deletion & 16,643 \\
Others & 22,212 \\
CDS & $3,455,409$ \\
C- gene segment & 41 \\
V- gene segment & 480 \\
cDNA match & 223,503 \\
Exon & $3,925,821$ \\
Gene & 186,625 \\
mRNA & 34,524 \\
\hline${ }^{*}$ CDS: Coding Sequence &
\end{tabular}

Copy Number Variation (CNV) is an important form of structural variation among individuals of the same species. The regions of the genome that have deleted or duplicated on some chromosomes between the sequencing individual and the reference correspond to CNVs. The CNV results listed in Table 5 and Figure 11 that is, over $72 \%$ of the CNV were down-regulated.

Table 5. Copy number variation (CNV) distribution and annotation distribution in the genome of Jordanian Awassi Sheep rams.

\begin{tabular}{lc}
\hline Copy number variation & Value \\
\hline CNV & 13,689 \\
Up-regulation & 3,743 \\
Down-regulation & 9,946 \\
CDS & 3,862 \\
C gene segment & 1 \\
V gene segment & 48 \\
cDNA match & 979 \\
exon & 5,070 \\
gene & 4,970 \\
mRNA & 11,987 \\
\hline *DS: Coding Sequence &
\end{tabular}

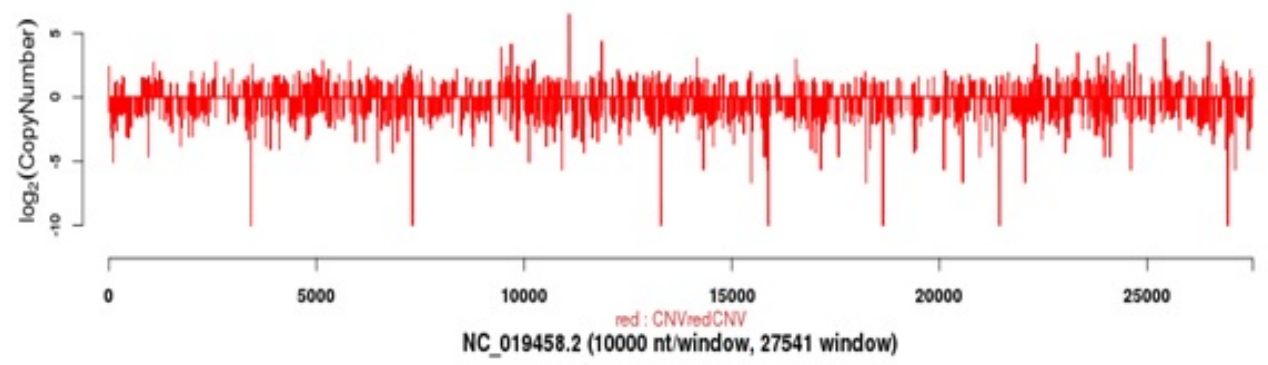

Figure 11. The distribution of copy number variations (CNVs) in genome NC_019458.2. 'Log 2 (Copy Number)' means a base-2 logarithmic copy number variation in each window.

\section{DISCUSSION}

In this project, we reportthe first Jordanian Awassi sheep whole-genome resequencing after the first simple sequencing (0.64-kb sequences) reported by (Al-Atiyat 
and Aljumaah, 2014; Jawasreh et al., 2019a and 2019b). Copy Number Variation (CNV) is an important form of structural variation among individuals of the same species. SNPs have a great impact on the variation of genomes and the biological traits, so it is helpful to look into the SNP annotations and paid particular attention to those in genic regions. Nonsynonymous SNPs believed to contribute to phenotypic variation among individuals or within populations (Jawasreh et al., 2019). They are also useful candidate mutations that may explain the different phenotypes among different animals.

Several SNPs that in different ovine genomic regions investigated and their associations with some economic Awassi sheep traits also tested. Jawasreh et al. (Jawasreh et al., 2017 and 2019) targeted a region between exons $61 \mathrm{bp} \mathrm{1C,} 88 \mathrm{bp} 1 \mathrm{D}$, and $473 \mathrm{bp}$ of the Awassi calpastatin gene and detected two non-synonymous variants that associated with growth performance and meat characteristics. Through simple sequencing procedure, Jawasreh et al. (2019) observed non-synonymous SNPs in the Awassi Beta-lactoglobulin, Prolactin, and Kappa Casein genes, the single effect and the interaction between the studied gene's SNPs genotypes analyzed and found to be of significant effect on Awassi milk production and composition. A non-synonymous mutation found in the Prolactin gene that associated with the birth weights of Awassi sheep explored (Jawasreh and Ismail, 2019a) and recommended using in selection to avoid dystocia. In the Awassi Calpastatin gene, individuals heterozygous in SNP were healthier than homozygous individuals (Jawasreh and Ismail, 2019b). It also associated the prolactin gene variants with prolificacy of Awassi sheep; BB genotype had 0.16 and 0.11 more lambs at birth than AA and AB, (Jawasreh et al., 2014). A SNP at base pair 34, 647, 499 on OAR23 detected through Genome-wide to show for Microtia in Awassi sheep (Jawasreh et al., 2016). Kijas et al. (2012) developed and used a set of 49,034 SNPs from a diverse collection of domestication and selection of sheep breeds in genotyping. The genome scan revealed 31 regions containing genes for coat pigmentation, skeletal morphology, body size, growth, and reproduction. They provide a high-density map of genetic variability viewed as an in-depth of the genetic history for sheep breed. Using Illumina Ovine 50K SNP BeadChip assay, Ahbara et al. (2019), identified putative candidate genes related to skeletal structure and morphology, fat deposition, and adaptation to environmental selection pressures in Ethiopian indigenous sheep. They suggested that Ethiopian fat-tail sheep could be a valuable animal genetic resource for understanding the genetic control of skeletal growth, fat metabolism, and associated physiological processes. Furthormore, to explore the genetic diversity and genetic structure of Ethiopian fat-tailed and Awassi sheep breeds. Using high density (50K) SNPs assay, Getachew et al. (2020), showed that the intense selection pressure on the Awassi sheep, resulted in a significant differentiation from local Awassi, and less withinpopulation variation and high level of runs of homozygosity based genomic inbreeding. They suggested using runs of homozygosity as a tool to control the level of inbreeding, which can help to widen the genetic base of the Ethiopian Awassi crossbreeding program.

The insertion or deletion of a particular region in the Ovine genome may cause overexpression or effect on some economic traits. Lassoued et al. (2017) reported a mutation in BMP15 gene called FecXBar in Barbarian sheep that associated with a single nucleotide substitution, deletion, and insertion this gene cDNA leading to a frameshift at protein position 101. This mutation affected the prolificacy of barbarian sheep and suggested using in selection for improving the prolificacy of this sheep breed. A nonmutant FecB mutation found in Awassi while they screened GDF9 gene in Awassi sheep and was 
polymorphic within the Awassi sheep breed without affecting Awassi ewes prolificacy (Jawasreh et al., 2014). In cattle, sheep, and pig, structural variation reported to linked to copy number variations (CNVs) for productive traits and diseases. SVs artificially selected in livestock such as a duplication of the agouti signaling protein gene that causes white coat color in sheep (Bickhart and Liu 2014).

In mammalian genomes, CNVs play a vital role in identifying their impact on natural and disease phenotypes. By using computational and experimental methods, it can catalog CNVs for critical functional characteristics with those in other mammalian genomes such as humans' genome (Guryev et al., 2008). Identifying genes based on the SNP variation requires additional validation for population structure. The potential of combining SNP variation and transcriptome data to identify putative genes that play a role in the improving of Awassi production or in the control of diseases that may affect sheep improvement.

\section{CONCLUSIONS}

The first whole Genome Resequencing of Jordanian Awassi Rams sheds light on various genetics and genomic studies. The results of this study focus in-depth on genetic variation, population structure, the distribution of (SNPs), the detected SNPs, and genetic variations in the whole genome could be used in marker assisted selection programs. Further investigations to determine the phylogeny, evolution, and gene pathways will be essential to expand our understanding of the evolutionary biology of economic traits such as milk, meat, wool production, and disease tolerance in Awassi sheep breeding and genetic improvement based on genomic selection.

\section{ACKNOWLEDGMENTS}

The authors are profoundly grateful to National Agricultural Research Center for their support, encouragement and for funding this project.

\section{CONFLICTS OF INTEREST}

The authors declare no conflict of interest.

\section{REFERENCES}

Ahbara A, Bahbahani H, Almathen F, et al. (2019). Genome-Wide Variation, Candidate Regions and Genes Associated With Fat Deposition and Tail Morphology in Ethiopian Indigenous Sheep. Front. Genet. 9: 699. doi:10.3389/fgene.2018.00699.

Al-Atiyat RM and Aljumaah RS (2014). Genetic distances and phylogenetic trees of different Awassi sheep populations based on DNA sequencing. Genet. Mol. Res. 13: 6557-6568.

Al-Atiyat RM, Tabbaa MJ, Salameh N and Tarawneh K (2012). Analysis of genetic variation of fat tailed-sheep in the southern region of Jordan. Asian J. Anim. Vet. Adv. 7: 378-389.

Al-Atiyat RM, Aljumaah RS, Alshaikh MA, et al. (2018). Microsatellite-Based Genetic Structure, and Diversity of Local Arabian Sheep Breeds. Front. Genet. 9: 408.

Barendse W, Vaiman D, Kemp SJ, Sugimoto Y, et al. (1997). A medium-density genetic linkage map of the bovine genome. Mamm. Genome. 8: 21-28.

Bickhart DM and Liu GE (2014). The challenges and importance of structural variation detection in livestock. Front. Genet. 5:37. 
Chen K, Wallis J, McLellan M, Larson D, et al (2009). BreakDancer: an algorithm for high-resolution mapping of genomic structural variation. Nat. Methods 6: 677.

Chessa B, Pereira F, Arnaud F, Amorim A, et al. (2009). Revealing the history of sheep domestication using retrovirus integrations. Science. 324(5926): 532-6.

Crawford AM, Dodds KG, Ede AJ, Pierson CA, et al. (1995). An autosomal genetic linkage map of the sheep genome. Genetics. 140: 703-724.

Getachew T, Haile A, Mészáros G, Rischkowsky B, et al. (2020). Genetic diversity, population structure and runs of homozygosity in Ethiopian short fat-tailed and Awassi sheep breeds using genome-wide 50k SNP markers. Livest. Sci. 232: 103899.

Guryev V, Saar K, Adamovic T, Verheul M, et al. (2008). Distribution and functional impact of DNA copy number variation in the rat. Nat. Genet. 40: 538.

Haddad N, Al-Nakeeb K, Petersen B, Dalen L, et al. (2017). Complete mitochondrial genome of the Oriental Hornet, Vespa orientalis F. (Hymenoptera: Vespidae). Mitochondrial DNA (B). 2(1): 139-140.

Haddad N, Batainh A. Migdadi O. Saini D, et al. (2016). Next-generation sequencing of Apis mellifera syriaca identifies genes for Varroa resistance and beneficial beekeeping traits. Insect Sci. 23 (4): 579-590.

Jawasreh K, Ababneh H, Awawdeh FT, Al -Massad MA, et al. (2012). Genotype and Allelic Frequencies of an Identified Mutation Causing Blindness in Jordanian Awassi Sheep Flocks. Asian Aust. J. Anim. Sci. 25(1): 33-36.

Jawasreh K, A babneh MM, Ismail ZB, Younes AM, et al. (2018). Genetic diversity and population structure of local and exotic sheep breeds in Jordan using microsatellite markers. Vet. World. 11(6): 778-781.

Jawasreh K, AlAmareen H and Pauline Y (2019). Relationships between Hha1 Calpastatin Gene Polymorphism, Growth Performance, and Meat characteristics of Awassi Sheep. Animals. 9: 667.

Jawasreh K and Al-Khasawneh AZ (2007). Genetic evaluation of milk production traits in Awassi sheep in Jordan. Egypt. J. Sheep Goat Sci. 12 (2): 83-100.

Jawasreh K, Al-Rawashdeh IM, Al-Majali A, Talafha H, et al. (2011). Genetic relatedness among Jordanian local Awassi lines Baladi, Sagri, and Blackface and the black Najdi breed using RAPD analysis. Genom. Quant. Genet. 2: 31-36.

Jawasreh K, Amareen AA and Aad P (2019). Effect and Interaction of $\beta$-Lactoglobulin, Kappa Casein, and Prolactin Genes on Milk Production and Composition of Awassi Sheep. Animals. 9(6): 382.

Jawasreh K, Awawdeh FT and Al-Qaisy A (2014). Association between GDF9, FecB, and Prolactin gene polymorphisms and prolificacy of Awassi sheep. Proceedings, 10th, World Congress of Genetics Applied to Livestock Production, Vancouver, BC, Canda, August 17-22, 2014.

Jawasreh K, Boettcher PJ and Stella A (2016). Genome-wide association scan suggests a basis for microtia in Awassi sheep. Animal Genet. 47(4): 504-6.

Jawasreh K, Hijazi J, Khasawneh A, Awawdeh F, et al. (2010). Quantitative and Molecular Genetic Analysis for Some Traits in Selected Jordanian Awassi Sheep For milk Production. World congress in on Genetics Applied to Livestock production. Leipzig, Germany.

Jawasreh K, Ismail ZB, Iya F, Castañeda-Bustos VJ, et al. (2018). Genetic parameter estimation for pre-weaning growth traits in Jordan Awassi sheep. Vet. World. 11(2): 254-258.

Jawasreh K and Ismail ZB (2019a). Polymorphism of prolactin, growth differentiation factor 9, and calpastatin genes and their effects on weight traits in Awassi lambs. J. Adv. Vet. Anim. Res. 6(1): 86-91.

Jawasreh K and Ismail ZB (2019b). Effects of calpastatin gene polymorphism on hematology and selected serum biochemical parameters in Awassi lambs. J. Adv. Vet. Anim. Res. 6(2): 193-6.

Jawasreh K, Jadallah R, Al-Amareen AH, Abdullah A, et al. (2017). Association between MspI calpastatin gene polymorphisms, growth performance, and meat characteristics of Awassi sheep. Indian J. Anim. Sci. 87(5): 635639.

Jiang Y, Xie M, Chen W, Talbot R, et al. (2014). The sheep genome illuminates the biology of the rumen and lipid metabolism. Science. 344: 6188.

Kijas JW, Lenstra JA, Hayes B, Boitard S, et al. (2012). Genome-wide analysis of the world's sheep breeds reveals high levels of historic mixture and strong recent selection. PLoS biology. 10(2): e1001258. doi:10.1371/journal.pbio.1001258.

Lassoued N, Benkhlil Z, Woloszyn F, Rejeb A, et al. (2017). FecXBar a Novel BMP15 mutation responsible for prolificacy and female sterility in Tunisian Barbarian Sheep. BMC Genet. 18: 43.

Li H and Durbin R (2009). Fast, and accurate short read alignment with Burrows-Wheeler Transform. Bioinformatics. 25: $1754-60$.

Maddox JF, Davies K, Crawford AM, Hulme DJ, et al. (2001). An Enhanced Linkage Map of the Sheep Genome Comprising More Than 1000 Loci. Genome Res. 11(7): 1275-1289.

McKenna A, Hanna M, Banks E, Sivachenko A, et al. (2010). The Genome Analysis Toolkit: a MapReduce framework for analyzing next-generation DNA sequencing data. Genome Res. 20: 1297-303.

Raadsma HW, Thomson PC, Zenger KR, Cavanagh C, et al. (2009). Mapping quantitative trait loci (QTL) in sheep. 1. A new male framework linkage map and QTL for growth rate and body weight. Genet. Sel. Evol. 41: 34. 
Tabbaa MJ (1998). The effect of age and sex on the body and fat-tail dimensions of Awassi sheep in Jordan. Mu'tah J. Res. Studies. 13: 63-74.

Tabbaa MJ (2003). Some factors are affecting the different body and fat-tail dimensions of Awassi ewes in Jordan. Dirasat Agric Sci. 30: 280-284.

Tabbaa MJ, Al-Azzawi WA and Campbell D (2001). Variation in fleece characteristics of Awassi sheep at different ages. Small Ruminant Res. 41: 95-100.

Tabbaa MJ, Alnimer MA, Shboul M and Titi HH (2008). Reproductive characteristics of Awassi ewes mated or to Jordanian or Syrian Awassi rams. Anim. Reprod. 5(1/2): 23-29.

Tabbaa MJ, Kridli RT, Amashe MG and Barakeh FS (2006). Factors Affecting Scrotal Circumference and Semen Characteristics of Awassi Rams. Jordan J. Agri. Sci. 2(3): 243 -250.

Wallberg A, Han F, Wellhagen G, Dahle B, et al. (2014). A worldwide survey of genome sequence variation provides insight into the evolutionary history of the honeybee Apis mellifera. Nat. Genet. 46(10): 1081-1090.

Zeder MA (2008). Domestication, and early agriculture in the Mediterranean Basin: origins, diffusion, and impact. Proc. Natl. Acad. Sci. U.S. A. 105: 11597-604.

Zheng L, Guo X, He B, Sun L, et al. (2011). Genome-wide patterns of genetic variation in sweet and grain sorghum (Sorghum bicolor). Genome Biol. 12: R114. 